\title{
Heart failure with preserved ejection fraction in the elderly
}

\section{Opinion}

Heart failure is a syndrome that derives from a structural or functional defect in the ventricular filling (heart failure with preserved ejection fraction, or HFPEF) or in the ejection of blood (heart failure with reduced ejection fraction). In this editorial, the preserved form, of this highly frequent disease in the elderly, will be approached with greater emphasis.

The definition of HFPEF has varied with the different methodologies adopted in previous studies. It is usually defined as a syndrome in which patients have symptoms and signs of heart failure with normal or near normal left ventricular ejection fraction (LVEF of more than $50 \%) .{ }^{1}$ This syndrome is clinically characterized by the presence of different types of dyspnea (on exertion, paroxysmal nocturnal or orthopnea), pulmonary rales, fatigue, elevated jugular venous pressure, lower extremity edema and exertion chest pain., ${ }^{2,3}$ Atrial fibrillation is also frequently seen and poorly tolerated in this cardiovascular condition, since the loss of atrial contraction can dramatically reduce left ventricular filling and limit the stroke volume. $^{4}$

Generally, patients with HFPEF are usually older, mainly women and have a previous history of hypertension. ${ }^{5}$ Obesity, coronary artery disease, diabetes mellitus, atrial fibrillation and hyperlipidemia are also highly prevalent in this form of heart failure in population-based studies and registries. ${ }^{1}$ However, hypertension remains the most important cause of this syndrome, with a prevalence of $60 \%$ to $89 \%$ in HFPEF patients. ${ }^{6}$

The ARIC study shows that this form of heart failure is by far the most dominant among older adults in the United States, representing $65 \%$ to $77 \%$ of prevalent cases. ${ }^{7}$ In comparison with heart failure of reduced ejection fraction, HFPEF patients are more likely to be older and to be females. These patients are also less likely to have had a prior myocardial infarction and a little less lower in-hospital mortality (3 versus 4 percent) but similar intensive care unit and hospital length of stay. ${ }^{8}$

Population aging and the increase in life expectancy of cardiac patients (thanks to therapeutic innovations) have led to a present increase in the prevalence of heart failure. A 2013 update from the American Heart Association estimated that there were 5.1 million people with this syndrome in the United States in $2006 .{ }^{9}$ The prevalence of heart failure with preserved ejection fraction steeply increases with age [10]. The Framingham Heart Study found a prevalence of heart failure in men of $0,8 \%$ at age 50 to 59 , increasing to $6,6 \%$ at ages 80 to 89 . Similar values $(0,8 \%$ and $7,9 \%)$ were noted in women. The prevalence in African-Americans is reported to be 25 percent higher than in white people. The incidence of this syndrome, just as its prevalence, increases with age. In this same study, the incidence approximately doubled over each successive decade of life, rising more steeply with age in women than in men. The annual incidence in men rose from $0,2 \%$ at age 35 to 64 to $1,2 \%$ at age 65 to $94 .{ }^{10}$
Volume I Issue 3 - 2017

\author{
Joao Lucas O Connell, Gabriela Carolina \\ Borges \\ Department of Cardiology, Federal University of Uberlandia, \\ Brazil
}

Correspondence: Joao Lucas O Connell, Department of Cardiology, Federal University of Uberlandia, Brazil, Tel 5534 99665-II59 Email oconnellj|@icloud.com

Received: May 01, 2017 | Published: May 10, 2017

The diagnosis is generally made by history and physical examination, echocardiography, and rarely by cardiac catheterization. ${ }^{11}$ The plasma brain natriuretic peptide or the N-terminal pro BNP may be used to consolidate the diagnosis, but a low level of these substances doesn't exclude the diagnosis. Among the complementary exams, the most important one is the echocardiography, which demonstrates the normal left ventricular ejection fraction and the defect in the ventricular filling. Other features that may be identified include left ventricular hypertrophy or concentric remodeling, left atrial enlargement, and evidence of diastolic dysfunction. ${ }^{12}$ A chest radiograph may show cardiomegaly and/or radiographic evidence of pulmonary edema. An electrocardiogram is routinely performed in patients with heart failure, although its findings are often nonspecific. Evidence of myocardial ischemia or prior infarction may be detected as well as signs of ventricular hypertrophy or left atrium enlargement.

The differential diagnosis includes cardiomyopathies, valve disease, and pericardial disease, right heart failure (including right ventricular infarction, arrhythmogenic right ventricular cardiomyopathy, and associated with pulmonary arterial hypertension not due to left ventricular disease).

The studies have demonstrated that the treatment normally used in heart failure with reduced ejection fraction (neurohumoral antagonists such as beta blockers, angiotensin converting enzyme inhibitors, angiotensin receptor blockers and cardiac resynchronization) do not decrease morbidity and mortality in the HFPEF patients. ${ }^{13}$ The treatment of this form of heart failure is based on the treatment of the contributing factors and comorbidities that are frequently present and that significantly impact the clinical course.

In general, management includes mineralocorticoid receptor antagonist and diuretics to treat volume overload. Other medications such as angiotensin receptor blockers, angiotensin converting enzyme inhibitors, calcium channel blockers, and beta-blockers are used as needed to treat hypertension but lack proven efficacy to alter clinical outcomes. The clinical efficacy of beta-blocker therapy, for example, was analyzed by the OPTIMIZE-HF, which compared the results in patients with heart failure with reduced ejection fraction and HFPEF patients. This study included 7154 elderly adults hospitalized 
with heart failure. In the group of reduced ejection fraction, betablocker therapy at hospital discharge was associated with reduced mortality and re-hospitalization rates. In contrast, among patients with preserved ejection fraction, this therapy was associated with lack of improvement in mortality or re-hospitalization.

Mortality in patients with preserved ejection fraction is lower when compared to patients with low ejection fraction, although survival was still much worse than it was in people without heart failure. Independent predictors of mortality in different studies included older age, male gender, New York Heart Association (NYHA) class III or IV, lower LVEF, extent of coronary artery disease, peripheral artery disease, diabetes, impaired renal function, and degree of diastolic dysfunction (as assessed by Doppler echocardiography). ${ }^{14,15}$

We can conclude that heart failure with preserved ejection fraction is a very frequent pathology in the elderly population, with a trend towards a progressive increase in its incidence and prevalence. $T$ The treatment normally used in heart failure with reduced ejection fraction (beta-blockers, angiotensin converting enzyme inhibitors, angiotensin receptor blockers) do not decrease morbidity and mortality in the HFPEF patients. The treatment of this form of heart failure is based on the treatment of the contributing factors and comorbidities that are frequently present, especially hypertension and myocardial ischemia. Therefore, it is important to develop new studies involving patients with this frequent disease, especially in geriatric population, in order to develop new therapies and preventive measures for the management of this complex syndrome.

\section{Acknowledgements}

None.

\section{Conflict of interest}

Author declares there is no conflict of interest in publishing the article.

\section{References}

1. Borlaug BA, Paulus WJ. Heart failure with preserved ejection fraction: pathophysiology, diagnosis, and treatment. Eur Heart J. 2011;32(6):670-679.

2. Paulus WJ, Tschöpe C. A novel paradigm for heart failure with preserved ejection fraction: comorbidities drive myocardial dysfunction and remodeling through coronary microvascular endothelial inflammation. $J$ Am Coll Cardiol. 2013;62(4):263-271.

3. Srivaratharajah K, Coutinho T, deKemp R, et al. Reduced Myocardial Flow in Heart Failure Patients With Preserved Ejection Fraction. Circ Heart Fail. 2016;9(7):pii:e002562.
4. Zakeri R, Chamberlain AM, Roger VL, et al. Temporal relationship and prognostic significance of atrial fibrillation in heart failure patients with preserved ejection fraction: a community-based study. Circulation. 2013;128(10):1085-1093.

5. Scantlebury DC, Borlaug BA. Why are women more likely than men to develop heart failure with preserved ejection fraction?. Curr Opin Cardiol. 2011;26(6):562-568.

6. Nohria A, Tsang SW, Fang JC, et al. Clinical assessment identifies hemodynamic profiles that predict outcomes in patients admitted with heart failure. J Am Coll Cardiol. 2003;41(10):1797-1804.

7. Shah AM, Claggett B, Loehr LR, et al. Heart Failure Stages Among Older Adults in the Community: The Atherosclerosis Risk in Communities Study. Circulation. 2017;135(3):224-240.

8. Yancy CW, Lopatin M, Stevenson LW, et al. Clinical presentation, management, and in-hospital outcomes of patients admitted with acute decompensated heart failure with preserved systolic function: a report from the Acute Decompensated Heart Failure National Registry (ADHERE) Database. J Am Coll Cardiol. 2006;47(1):76-84.

9. Go AS, Mozaffarian D, Roger VL, et al. Heart disease and stroke statistics--2013 update: a report from the American Heart Association. Circulation. 2013;127(1):e6-e245.

10. Ho KK, Pinsky JL, Kannel WB, et al.The epidemiology of heart failure: the Framingham Study. J Am Coll Cardiol. 1993;22(4):6-13.

11. Paulus WJ, Tschöpe C, Sanderson JE, et al. How to diagnose diastolic heart failure: a consensus statement on the diagnosis of heart failure with normal left ventricular ejection fraction by the Heart Failure and Echocardiography Associations of the European Society of Cardiology. Eur Heart J. 2007;28(20):2539-2550.

12. Andersen MJ, Borlaug BA. Heart failure with preserved ejection fraction: current understandings and challenges. Curr Cardiol Rep. 2014;16(7):501.

13. Schwartzenberg S, Redfield MM, From AM, et al. Effects of vasodilation in heart failure with preserved or reduced ejection fraction implications of distinct pathophysiologies on response to therapy. J Am Coll Cardiol. 2012;59(5):442-451.

14. Hernandez AF, Hammill BG, O'Connor CM, et al. Clinical effectiveness of beta-blockers in heart failure: findings from the OPTIMIZE-HF (Organized Program to Initiate Lifesaving Treatment in Hospitalized Patients with Heart Failure) Registry. J Am Coll Cardiol. 2009;53(2):184-192.

15. Jones RC, Francis GS, Lauer MS. Predictors of mortality in patients with heart failure and preserved systolic function in the Digitalis Investigation Group trial. J Am Coll Cardiol; 2004;44(5):1025-1029. 